\title{
Revealing Large-Scale Homogeneity and Trace Impurity Sensitivity of GaAs Nanoscale Membranes
}

Z. Yang, ${ }^{\dagger}$ A. Surrente, ${ }^{\dagger}$ G. Tutuncuoglu, ${ }^{\ddagger}$ K. Galkowski, ${ }^{\dagger, \S}$ M. Cazaban-Carrazé, ${ }^{\dagger}$ F. Amaduzzi, ${ }^{\ddagger}$ P. Leroux, D. K. Maude, ${ }^{\dagger}$ A. Fontcuberta i Morral, ${ }^{*}+\underset{\text { and P. Plochocka* }}{* \dagger}+0$

${ }^{\dagger}$ Laboratoire National des Champs Magnétiques Intenses, CNRS-UGA-UPS-INSA, 143 avenue de Rangueil, 31400 Toulouse, France

${ }^{\ddagger}$ Laboratory of Semiconductor Material, École Polytechnique Fédérale de Lausanne, 1015 Lausanne, Switzerland

${ }^{\S}$ Institute of Experimental Physics, Faculty of Physics, University of Warsaw, Pasteura 5, 02-093 Warsaw, Poland

Supporting Information

ABSTRACT: III-V nanostructures have the potential to revolutionize optoelectronics and energy harvesting. For this to become a reality, critical issues such as reproducibility and sensitivity to defects should be resolved. By discussing the optical properties of molecular beam epitaxy (MBE) grown GaAs nanomembranes we highlight several features that bring them closer to large scale applications. Uncapped membranes exhibit a very high optical quality, expressed by extremely narrow neutral exciton emission, allowing the resolution of the more complex excitonic structure for the first time. Capping of the membranes with an AlGaAs shell results in a strong increase of emission intensity but also in a shift and broadening of the exciton peak. This is attributed to the existence of impurities in the shell, beyond MBE-grade quality, showing the high sensitivity of these structures to the presence of impurities. Finally, emission properties are identical at the submicron and submillimeter scale, demonstrating the potential of these structures for large scale applications.

KEYWORDS: GaAs/AlGaAs nanomembranes, photoluminescence,

electronic and optical properties of ensemble vs single nanomembrane
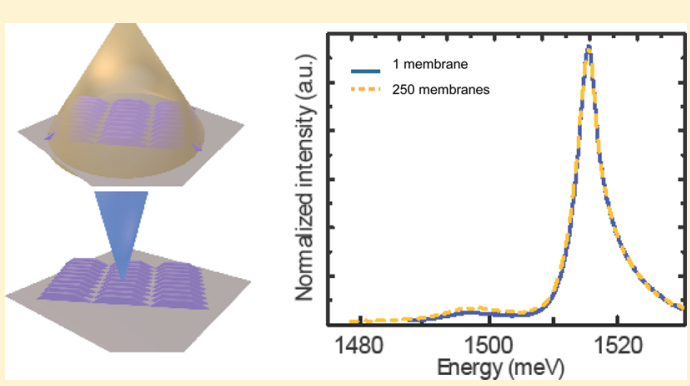

T anowires (NWs) are filamentary crystals with a diameter 1 in the submicrometer down to nanometer range. Their special morphology, dimensions and high surface-to-volume ratio are often translated into advantageous optical and electrical properties. As a consequence, they have been widely used in electronics, ${ }^{1-5}$ optoelectronics, ${ }^{6,7}$ solar cells, ${ }^{8-11}$ and sensors. ${ }^{12,13}$ If not adequately passivated, the surface recombination can limit the optical performance of the NWs. ${ }^{14}$ In addition, surface depletion can also affect the volume distribution and separation of the carriers in the NW. ${ }^{15-19}$ Different passivation methods have been employed in the past, notably capping of the free surfaces with a higher bandgap shell around the nanowire. ${ }^{20-22}$ Nevertheless, capping also modifies the nature of the surface. Several effects have been reported, such as band bending at the interface leading to the accumulation of the charge at the interface or piezoelectric strain. ${ }^{23-27}$ In addition, the AlGaAs alloy typically used for capping GaAs nanowires is generally inhomogeneous, with directed and random segregation of $\mathrm{Ga}$ and $\mathrm{Al}$ forming, respectively, Al-rich ridges and Ga-rich nanoscale islands. ${ }^{28,29}$ Simultaneously, III-V NWs can suffer from twin defects and polytypism, ${ }^{30,31}$ which adversely affect their electronic and optical properties. ${ }^{32-34}$ With a judicious optimization of growth conditions, single NW with a pure zinc-blende or wurzite structure can be obtained. ${ }^{35-37}$ Still, the optical and electronic properties tend to fluctuate considerably from NW to NW, which precludes the proper control of the response of an ensemble of nanowires.

Recently, alternative approaches to obtain defect-free nanostructures have been proposed. Particularly promising is the inversion of polarity from B to A as well as template assisted and nanomembrane assisted selective epitaxy (TASE and MASE, respectively). All these techniques provide defect free III-V nanostructures by blocking the formation of twinning defects. ${ }^{38-43}$ An additional advantage of these approaches is the possibility to engineer the shape, so that membranes, ${ }^{43}$ sails, ${ }^{42}$ or sheets ${ }^{39,41}$ can be grown. Nanoscale membranes show relatively long minority carrier diffusion length of $180 \mathrm{~nm}$ at 4.2 $\mathrm{K}$, which is significantly larger than the diffusion lengths found in nanowires. ${ }^{40,41}$ Moreover, by introducing passivation and/or doped structures, the design can be further sophisticated. ${ }^{40,41}$ The transfer of NW optoelectronic devices to industry requires achieving highly reproducible and uniform structures through a large surface area, so that the properties of ensemble and single object are indistinguishable. For instance, in photoluminescence this implies indistinguishability in terms of line width and

Received: January 19, 2017

Revised: April 11, 2017

Published: April 25, 2017 
emission energy and spectral shape. Growing the nanostructures using TASE and MASE turned out to be the most promising direction to achieve large area highly uniform systems.

In this work we demonstrate, by using optical techniques, that GaAs nanoscale membranes provide the settings for extremely high quality nanostructures, both from the structural and functional point of view. We elucidate how the improvement in functional properties is homogeneous across the whole wafer. This shows the potential of these nanostructures for nanotechnology and opens the path toward large scale nanomanufacturing. Furthermore, we provide very strong evidence that capping of the membranes, despite increasing the emission efficiency, unexpectedly leads to the degradation of their optical properties.

Nanomembranes have been grown using selective area epitaxy (for growth details see methods and ref 43). In Figure la, a tilted SEM image of a GaAs nanomembrane array
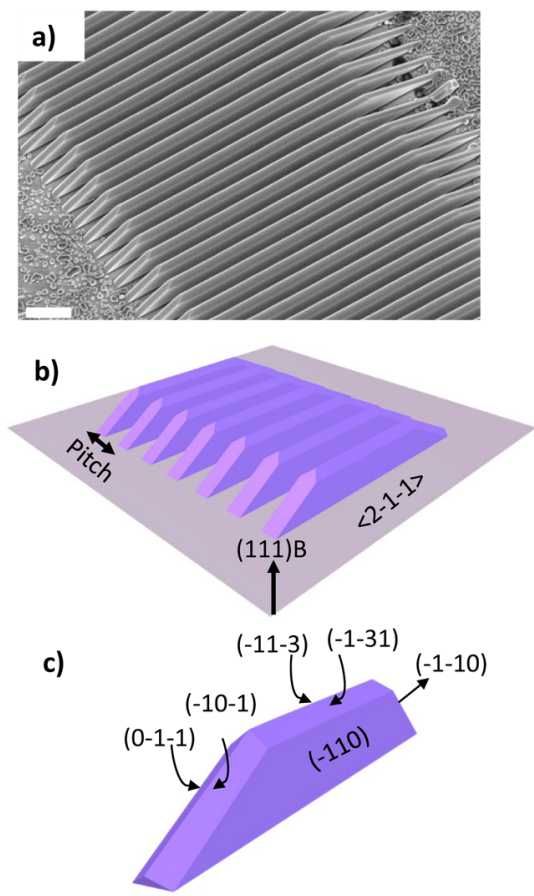

Figure 1. (a) SEM image ( $20^{\circ}$ tilted) of a GaAs nanomembrane array. (b) Three-dimensional model of the membrane array signifying the orientation of the structures. (c) Faceting of a GaAs membrane.

consisting of $10 \mu \mathrm{m}$ long and $100 \mathrm{~nm}$ wide nanomembranes with $500 \mathrm{~nm}$ pitch, used in the further optical experiments, are shown. Pitch is defined as the distance between the membranes, as depicted in Figure $1 \mathrm{~b}$. Nanomembranes are oriented in $\langle 2 \overline{11}\rangle$ direction, which is perpendicular to (111) B and ( $\overline{110})$ directions and expose the facets shown in Figure 1c. Most of the facets belong to $\{110\}$ family except high index top facets of $(\overline{1} 1 \overline{3})$ and $(\overline{13} 1)$. Adjusting the membrane orientation to $\langle 2 \overline{11}\rangle$ and growth conditions, it is possible to obtain pure zinc-blende structures with high-aspect ratio with molecular beam epitaxy (MBE). ${ }^{43}$ Detailed growth conditions are given in the methods section. The reported shape is the result of an hour growth with $1 \AA / s$ growth rate. If growth is continued long enough, the morphology of the membrane evolves into a triangular shape. During the growth of AlGaAs shell the $(\overline{110})$ facet transforms to $(\overline{22} 1)$.
Typical normalized $\mu \mathrm{PL}$ spectra of a single uncapped GaAs and capped $\mathrm{GaAs} / \mathrm{Al}_{x} \mathrm{Ga}_{1-x}$ As nanomembranes are presented in Figure 2a. For the capped membranes the data have been taken for three different compositions of the shell $(x=15,33$, $50 \%)$. Overall, the emission spectra are composed of two bands, around 1515 and $1490 \mathrm{meV}$. The higher energy band corresponds to the band-edge luminescence of the GaAs membranes, while the lower energy emission can be attributed to the donor-acceptor transitions due to carbon impurities normally present in commercial GaAs substrates, ${ }^{44,45}$ which was further observed in detailed cathodoluminescence studies. Our spectra are comparable to previously reported optical emission in nanomembranes with $33 \% \mathrm{Al}$ composition in the shell. ${ }^{43}$ The peak related to the carbon impurities can be used as a reference for the luminescence intensity. After capping, the emission from the GaAs membrane increases dominating the carbon related PL. The dramatic increase of the emission from the membrane is a direct consequence of the surface passivation that reduces the nonradiative surface recombination.

The detailed nature of the emission is very different for capped and uncapped samples. For uncapped membranes the spectrum is composed of three lines (see Figure2b). The peak at the highest energy of $\sim 1515.5 \mathrm{meV}$ corresponds to the free exciton emission, while the two peaks at lower energies are related to neutral donor bound exciton emission $\left(D^{0}-X\right)$ and acceptor bound exciton emission $\left(A^{0}-X\right)$ with emission energies that are typical for bulk GaAs. ${ }^{46,47}$ This result rules out any possible quantum confinement in the nanomembranes. This is not unexpected since the exciton Bohr radius of $\simeq 14 \mathrm{~nm}$ in GaAs is much smaller than the size of the membrane. ${ }^{48}$ In contrast, the typical emission spectra for the GaAs nanomembranes capped with $\mathrm{Al}_{x} \mathrm{Ga}_{1-x}$ As layer (Figure $2 \mathrm{~b}$ ) are composed of a single line, which we attribute to the neutral exciton recombination. Emission lines from $D^{0}-X$ and $A^{0}-X$ are completely absent. The neutral exciton emission energy red shifts and broadens with increasing $\mathrm{Al}$ shell content. To quantify this effect we have measured the power dependence of the energy and full width at the half-maximum (fwhm) of the neutral exciton emission. In Figure $2 c$ the emission energy is plotted as a function of excitation power. For membranes with high aluminum shell content $(x \geq 0.3)$ a blue shift is observed with increasing excitation power, which quickly saturates for powers above a few $\mu \mathrm{W}$. For powers of $10 \mu \mathrm{W}$ and above the emission energy is independent of the excitation power. There is a clear and systematic decrease in the emission energy (redshift) with increasing $\mathrm{Al}$ content. This is illustrated in the inset in the Figure $2 c$, where the energy difference between uncapped and capped emission $\Delta E$ is plotted as a function of the shell aluminum composition $x$ for the same excitation power. In Figure $2 \mathrm{~d}$ the fwhm of the emission is plotted as a function of the excitation power. The line widths increase slightly with increasing power, but this is negligible compared to the increase in the fwhm with increasing Al content of the shell. In the inset of Figure $2 \mathrm{~d}$ we plot the fwhm versus the shell $\mathrm{Al}$ content for an excitation power of $10 \mu \mathrm{W}$. The line width is multiplied by roughly a factor of 5 between the uncapped membrane and the membrane with a $50 \% \mathrm{Al}$ content cap layer. Thus, while capping the membranes reduces nonradiative surface recombination, leading to enhanced neutral exciton emission, it also detrimentally affects the optical properties of the GaAs core, leading to a significantly broadened emission.

We turn now to the effect of the red-shift of the excitonic emission upon capping the membranes with AlGaAs. In fact, a 

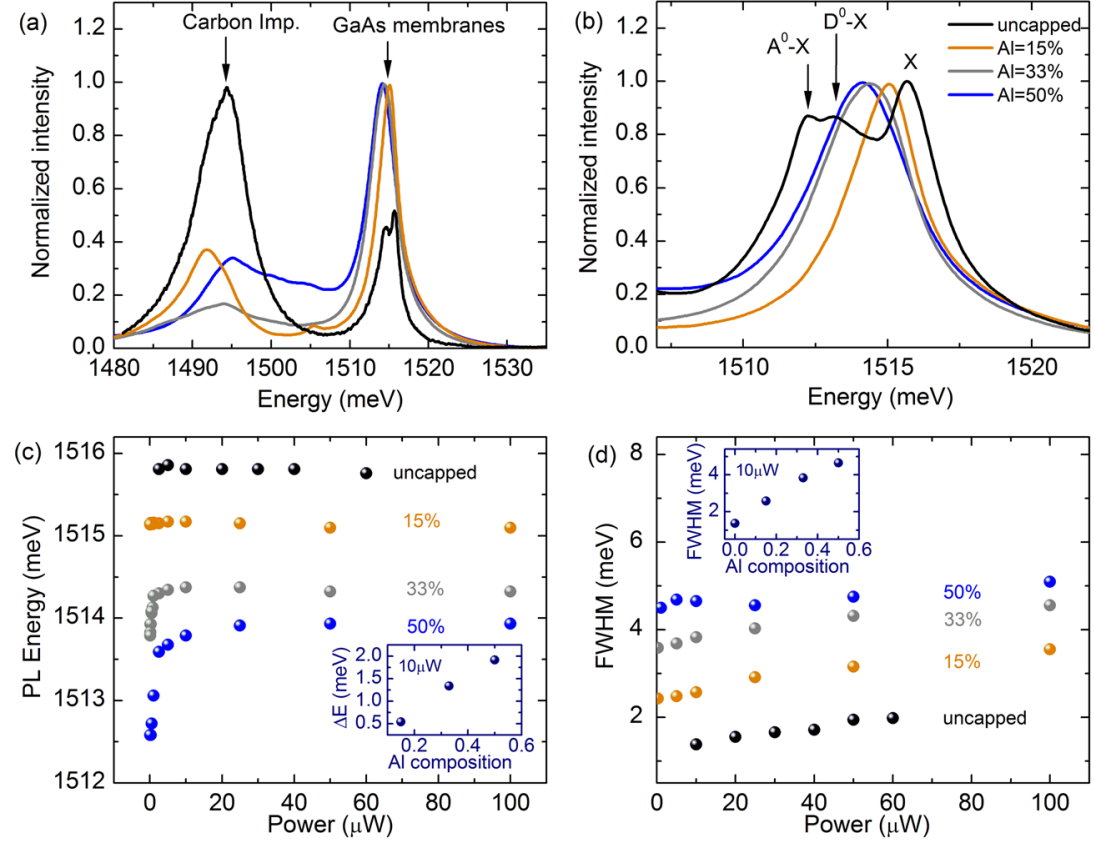

Figure 2. Normalized photoluminescence spectra of the uncapped GaAs and capped GaAs/AlGaAs nanomembranes in a (a) wide (full) and (b) narrower (emission from the core) energy range. (c) Emission energy and (d) fwhm of the free exciton emission as a function of the excitation power. Red-shift of the PL for different Al composition is plotted as symbols in the inset in panel (c). The inset in panel (d) shows the evolution of the fwhm of the PL line for different $\mathrm{Al}$ composition in the shell.

similar effect has been observed previously for a simple $\mathrm{AlGaAs} / \mathrm{GaAs}$ interface, ${ }^{23,49} \mathrm{InP}$ nanowires, ${ }^{50}$ and GaAs nanowires capped with AlGaAs shell. ${ }^{24,26,27}$ For simple $\mathrm{AlGaAs} / \mathrm{GaAs}$ the band bending at the interface forms a pocket for the electrons or holes. ${ }^{23,49}$ Such confined carriers at the interface will recombine with the free carriers (of the opposite species) in the valence or conduction band at a sufficient distance from the interface that flat-band conditions have been re-established. As the charges are spatially separated, emission has a spatially indirect character and is red-shifted in comparison to the simple excitonic emission observed in uncapped GaAs. Moreover, the band bending can be screened by photocreated carriers decreasing the overall effect with the increase of the excitation power. For InP nanowires a similar picture has been proposed, where the band bending was induced by a pinning of the Fermi level. ${ }^{50}$ Finally, for GaAs nanowires capped with AlGaAs shell, the mechanism of the band bending can be enriched by strain, related to the shell thickness. ${ }^{26,27}$ However, the strain plays a significant role only for rather thick shells. In the case of the nanomembranes the core is much thicker than the shell. Additional confirmation of the negligible role of the strain in our structures is given by the Raman spectroscopy. If the shift we observe originated from strain, it would imply a significantly lower Al composition than the nominal composition. ${ }^{51}$ Our Raman measurements (see SI) confirm that the $\mathrm{Al}$ composition corresponds very well to the nominal composition in the nanomembranes and the lack of strain in the membrane core.

We attribute the observed red shift of the emission to the indirect nature of the exciton recombination at the capping interface. Due to residual doping in the AlGaAs shell, band bending occurs at the AlGaAs/GaAs interface. To this end, we illustrate in Figure $3 \mathrm{a}$ the position of the valence and conduction band edges as a function of the distance from the membrane surface. Our hypothesis is that the AlGaAs shell
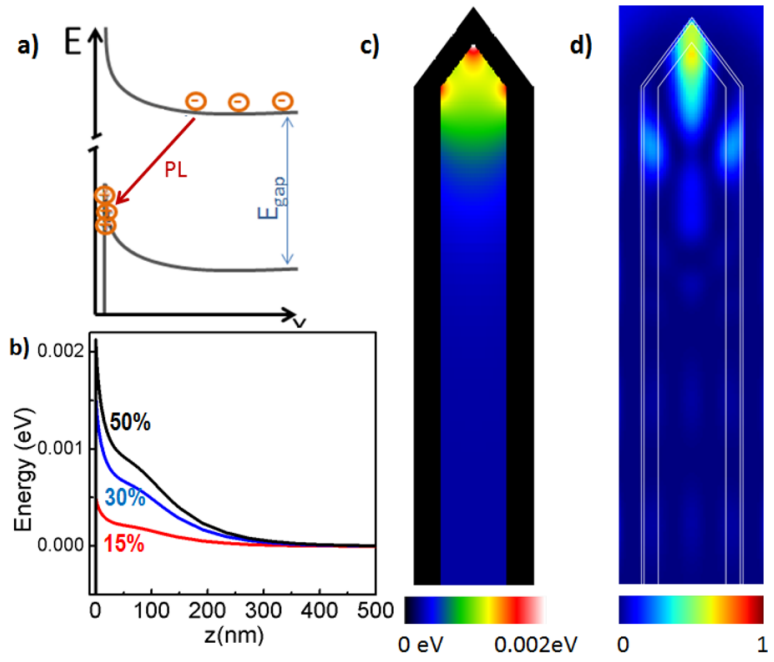

Figure 3. (a) Position of the valence and conduction band edges as a function of the distance from the membrane surface. (b) Band bending at the tip of the membrane as a function of the distance to the surface and for the three $\mathrm{Al}$ contents. (c) Two-dimensional valence and conduction band maps for an $\mathrm{Al}$ concentration of 50\%. (d) Crosssectional map of the electric field energy density for a nanomembrane under the presence of a monochromatic wave coming from the top and with parallel polarization.

contains some oxygen impurities, associated with the addition of aluminum. Secondary ion mass spectroscopy measurements on $\mathrm{AlGaAs}$ layers indeed indicate a slight O-contamination associated with aluminum (see SI). This contamination is still better than the purity specifications of MBE-grade aluminum, $6 \mathrm{N5}^{\prime}$, which implies that nanostructures are much more sensitive than bulk structures to impurities. Thus, the optical response of high quality nanostructures provides a sensitive means to detects extremely low levels of impurities. The red- 
shift of the luminescence at high excitation powers is larger for higher $\mathrm{Al}$ content (see Figure $3 \mathrm{a}$ ). This shows that the band bending increases with the $\mathrm{Al}$ content in the shell as the exciton recombination becomes more indirect.

Our observations are further supported by the simulation of the band bending at the AlGaAs/GaAs interface by solving Poisson and Schrödinger equations self-consistently with the software nextnano3. In the model we have included the presence of p-type interface states between GaAs and AlGaAs shell, which increases with increasing $\mathrm{Al}$ content. Our experimental data fits well with $2 \times 10^{9}, 6 \times 10^{9}$, and $8 \times$ $10^{9} \mathrm{~cm}^{-2}$ interface dopants for an $\mathrm{Al}$ concentration of $15 \%$, $33 \%$, and $50 \%$, respectively. Figure $3 \mathrm{~b}$ shows the resulting band bending at the tip of the membrane as a function of the distance to the surface and for the three $\mathrm{Al}$ contents. Here is evident the presence of a triangular potential in the valence band at the interface GaAs/AlGaAs where holes can be trapped. We can also observe an increase of the height of the potential with $\mathrm{Al}$ content, which results in a red-shift of the indirect transition.

It is worth noting that the red shift observed in our samples is of comparable magnitude with that observed by Songmuang at $\mathrm{al}^{26}$ but much smaller than that reported by Dhaka et al. ${ }^{24}$ This discrepancy can be partly ascribed to the MetalOrganic Vapor Phase Epitaxy (MOVPE) employed by Dhaka et al. ${ }^{24}$ to grow their nanowires. MOVPE involves the use of metalorganic species as group III precursors, which might introduce an unintentionally high concentration of impurities.

The small blue shift observed at low powers, which saturates around $10 \mu \mathrm{W}$, has been also observed for GaAs nanowires capped with $\mathrm{AlGaAs}$ shell, ${ }^{26,27}$ and it was associated with the presence of some negatively charged traps at the interface, which are filled by photocreated carriers in the AlGaAs shell, that migrate toward interface. Once filled, they can no longer modify the band bending at the interface, which explains the saturation of the blue-shift of the emission energy above 10 $\mu \mathrm{W}$, indicating that the band bending is the dominant effect in our nanomembranes.

The special geometry of the nanoscale membranes requires some further modeling. First, the nonflat geometry of the interface should result into a spatially dependent band bending. In addition, the vertical nature of the membranes can additionally lead to nonhomogeneous light absorption. ${ }^{28}$ Figure $3 \mathrm{c}$ shows the $2 \mathrm{D}$ valence and the conduction band maps for an $\mathrm{Al}$ concentration of $50 \%$. We can observe a band-bending at the interface, which is significantly larger at the top corner of the nanomembrane. We have simulated the electromagnetic field distribution using the package Meep, a freely available software implementing the Finite Difference in Time Domain Method ${ }^{52}$ taking into consideration the exact geometry of the core/shell nanomembrane with a shell of $33 \%$ of $\mathrm{Al}$. The dielectric constant is taken from ref 53. Figure $3 \mathrm{~d}$ shows the crosssectional map of the computed electric field energy density for a nanomembrane under the presence of a monochromatic wave coming from the top and with parallel polarization. It is clearly seen that the field energy is not distributed evenly across the cross-section but is rather confined at the top edge of the nanomembrane. This means that our $\mu$ photoluminescence experiments mainly probe the exciton properties at the tip, where the band bending is more pronounced. The results of this simulation explains also the broadening of the emission with the increasing $\mathrm{Al}$ content. Although emission is probed locally, the probed region can contain inhomogeneity band bending leading to the broadening of the emission peak. This is in perfect agreement with the observation that the effect is the strongest for the highest $\mathrm{Al}$ composition.

Finally, we come to perhaps the most striking and novel property of these nanomembranes, namely, their reproducibility and large scale uniformity. While epitaxial MBE provides highly uniform growth, this is not the case for the self-organized growth of quantum dots or NWs, where nucleation events in growth follow Poissonian statistics that lead to a distribution in the properties. As an example, in NWs this leads to a twinning or stacking fault density that varies from NW to NW (complete defect-free structures are rare). As a result, the optical properties vary from NW to NW and macro-photoluminescence measurements of the ensemble normally do not match microphotoluminescence of a single NW. We have recently shown that MBE growth using selective area epitaxy can produce arrays of defect free nanomembranes. ${ }^{43}$ However, optical investigations were limited to PL of a single nanomembrane so that the uniform optical properties of an ensemble has never been demonstrated.

To demonstrate large scale uniformity, we compare the emission spectra of a single membrane with the ensemble emission of around 250 membranes measured using macro-PL, achieved here by defocusing the laser spot. Representative PL spectra are shown in Figure 4 for the capped and uncapped
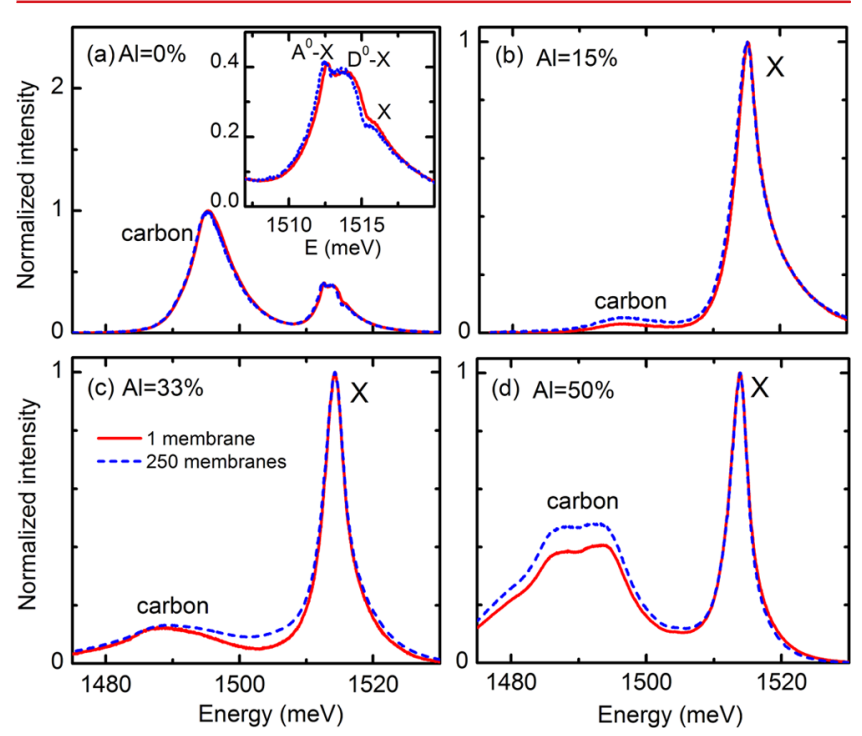

Figure 4. $(a-d)$ Normalized photoluminescence spectra of the uncapped GaAs and capped GaAs/AlGaAs nanomembranes measured at low temperature. The solid red and dashed blue line correspond to the excitation/emission from 1 and 250 membranes, respectively. The inset shows the zoom of the GaAs core emission of the uncapped membranes.

membranes. Defocusing increases the contribution of the substrate, which is reflected in the slightly increased amplitude of the carbon related emission seen in Figure 4. The substrate $\mathrm{PL}$ is dominated by the carbon related emission, and free exciton emission is not observed from the substrate. We have mapped the luminescence properties of the membranes by cathodoluminescence in a previous work. ${ }^{43}$ These measurements confirm that the carbon-related peak originates solely from the substrate.

Surprisingly, the PL originating from single membrane is almost identical to the ensemble emission. The carbon impurity 
emission is slightly enhanced in the ensemble emission of the capped samples ( $\simeq 20 \%$ for the $50 \% \mathrm{Al}$ membrane). This is probably due to the inhomogeneous distribution of the carbon impurities across the substrate. In contrast, the neutral exciton emission is strictly identical in both the energy of the emission and the line width for all samples. In the uncapped sample, the neutral and bound exciton emission is also almost indistinguishable (see inset Figure 4a). The identical emission from a single and an ensemble of membranes unequivocally demonstrates the very high quality of the crystal structure and extremely high reproducibility of the nanomembranes, which has never been observed for the classical radial nanowires. Moreover, data measured at different places on the same membrane, and on different membranes, vary very little in intensity, energy position, or line width, suggesting an excellent crystal quality of the uncapped membranes.

In conclusion, we have demonstrated luminescence properties of GaAs membranes that are on a par with the best twodimensional layers obtained with MBE. Upon capping of the membranes with an AlGaAs layer the PL emission is strongly enhanced but also unexpectedly accompanied by a degradation of the optical properties with a significant broadening of the exciton emission. Capping also leads to a red shift of the emission which has been attributed to the residual carbon doping of Al-containing layers which leads to band bending at the AlGaAs/GaAs interface. The quality of the membrane growth process is further supported by ensemble measurements, which are almost indistinguishable from the single membrane results. Additionally, our results show an extreme high sensitivity of the optical response of the nanomembranes on impurity concentration that goes beyond what is possible in terms of state of the art high purity MBE.

Methods. GaAs nanomembranes used in that study are grown with a DCA solid source molecular beam epitaxy (MBE) system. Substrates are PECVD deposited $\mathrm{SiO}_{2}$ masked (111) B GaAs. The oxide thickness is $30 \mathrm{~nm}$. The growth mask is patterned with a combination of e-beam lithography and fluorine based dry etching as reported earlier. ${ }^{43}$ The growth temperature is $635^{\circ} \mathrm{C}$, the growth rate is $1 \AA / s$, and the V/III ratio is 10 for the GaAs core. The length of nanomembranes and the distance between them are defined by patterning the $\mathrm{SiO}_{2}$ mask. We focused our characterization on structures with $100 \mathrm{~nm}$ width, $500 \mathrm{~nm}$ pitch, and $10 \mu \mathrm{m}$ length. In the case of capped GaAs nanomembranes, the structures are capped with a shell of $\mathrm{Al}_{x} \mathrm{Ga}_{1-x} \mathrm{As}(x=0.15,0.3$ and 0.5$)$. The substrate temperature is reduced to $460{ }^{\circ} \mathrm{C}$, and As flux is increased to 1 $\times 10^{-5}$ Torr for shell growth. Nominal thickness of AlGaAs shell is always $50 \mathrm{~nm}$, and it is protected with $10 \mathrm{~nm}$ of GaAs against oxidation. Aluminum ratios and nominal AlGaAs layer thicknesses are deduced from reflection high-energy electron diffraction (RHEED) calibrations performed on (100) GaAs substrates.

For the measurements the samples were placed in a helium flow cryostat with optical access. The cryostat was mounted on the motorized $x-y$ translation stages, which allow high resolution spatial mapping. A microscope objective $50 \times$ with a numerical aperture NA $=0.55$ was used to focus the excitation beam and collect the PL from the nanomembranes. The laser spot could be focused down to a diameter of $\simeq 0.5 \mu \mathrm{m}$ (diffraction limit), which enabled us to optically address single (or a maximum of two in a worst case scenario) membranes. To investigate many membranes, the laser spot was defocused. The steady-state $\mu \mathrm{PL}$ signal was excited with a $532 \mathrm{~nm}$ laser, and the spectra were recorded using a spectrometer equipped with a liquid nitrogen cooled CCD camera. All the measurements presented here have been performed at $T=4.2 \mathrm{~K}$.

\section{ASSOCIATED CONTENT}

\section{Supporting Information}

The Supporting Information is available free of charge on the ACS Publications website at DOI: 10.1021/acs.nanolett.7b00257.

Additional characterization of membranes, inelastic light scattering (Raman), and secondary ion mass spectroscopy (SIMS) profile (PDF)

\section{AUTHOR INFORMATION}

\section{Corresponding Authors}

*E-mail: anna.fontcuberta-morral@epfl.ch.

*E-mail: paulina.plochocka@lncmi.cnrs.fr.

ORCID

P. Plochocka: 0000-0002-4019-6138

Notes

The authors declare no competing financial interest.

\section{ACKNOWLEDGMENTS}

This work was partially supported by ANR JCJC project milliPICS, the Région Midi-Pyrénées under contract MESR 13053031, and BLAPHENE project under IDEX program Emergence.

\section{REFERENCES}

(1) Hu, J.; Odom, T. W.; Lieber, C. M. Acc. Chem. Res. 1999, 32, $435-445$.

(2) Cui, Y.; Lieber, C. M. Science 2001, 291, 851.

(3) Thelander, C. Mater. Today 2006, 9, 28.

(4) Lieber, C. M.; Wang, Z. L. MRS Bull. 2007, 32, 99-108.

(5) Lu, W.; Lieber, C. M. Nat. Mater. 2007, 6, 841.

(6) Hua, B.; Motohisa, J.; Kobayashi, Y.; Hara, S.; Fukui, T. Nano Lett. 2009, 9, 112-116. PMID: 19072060.

(7) Saxena, D.; Mokkapati, S.; Parkinson, P.; Jiang, N.; Gao, Q.; Tan, H. H.; Jagadish, C. Nat. Photonics 2013, 7, 963-968.

(8) Atwater, H. A.; Polman, A. Nat. Mater. 2010, 9, 205-213.

(9) Wallentin, J.; Anttu, N.; Asoli, D.; Huffman, M.; Åberg, I.; Magnusson, M. H.; Siefer, G.; Fuss-Kailuweit, P.; Dimroth, F.; Witzigmann, B.; Xu, H. Q.; Samuelson, L.; Deppert, K.; Borgström, M. T. Science 2013, 339, 1057-1060.

(10) Krogstrup, P.; Jørgensen, H. I.; Heiss, M.; Demichel, O.; Holm, J. V.; Aagesen, M.; Nygard, J.; Fontcuberta i Morral, A. Nat. Photonics 2013, 7, 306-310.

(11) Mann, S. A.; Oener, S. Z.; Cavalli, A.; Haverkort, J. E. M.; Bakkers, E. P. A. M.; Garnett, E. C. Nat. Nanotechnol. 2016, 11, 1071.

(12) Tian, B.; Liu, J.; Dvir, T.; Jin, L.; Tsui, J. H.; Qing, Q.; Suo, Z.; Langer, R.; Kohane, D. S.; Lieber, C. M. Nat. Mater. 2012, 11, 986994.

(13) Takei, K.; Takahashi, T.; Ho, J. C.; Ko, H.; Gillies, A. G.; Leu, P. W.; Fearing, R. S.; Javey, A. Nat. Mater. 2010, 9, 821-826.

(14) Nelson, R. J.; Sobers, R. G. Appl. Phys. Lett. 1978, 32, 761-763.

(15) Schricker, A. D.; Davidson, F. M., III; Wiacek, R. J.; Korgel, B. A. Nanotechnology 2006, 17, 2681.

(16) Parkinson, P.; Lloyd-Hughes, J.; Gao, Q.; Tan, H. H.; Jagadish, C.; Johnston, M. B.; Herz, L. M. Nano Lett. 2007, 7, 2162-2165.

(17) Demichel, O.; Heiss, M.; Bleuse, J.; Mariette, H.; Fontcuberta i Morral, A. Appl. Phys. Lett. 2010, 97, 201907.

(18) Katzenmeyer, A. M.; Léonard, F.; Talin, A. A.; Wong, P. S.; Huffaker, D. L. Nano Lett. 2010, 10, 4935-4938. PMID: 21053980.

(19) Calarco, R.; Marso, M.; Richter, T.; Aykanat, A. I.; Meijers, R.; v.d. Hart, A.; Stoica, T.; Lüth, H. Nano Lett. 2005, 5, 981-984. 
(20) Noborisaka, J.; Motohisa, J.; Hara, S.; Fukui, T. Appl. Phys. Lett. 2005, 87, 093109.

(21) Chang, C. C.; Chi, C.-Y.; Yao, M.; Huang, N.; Chen, C. C.; Theiss, J.; Bushmaker, A. W.; LaLumondiere, S.; Yeh, T. W.; Povinelli, M. L.; Zhou, C.; Dapkus, P. D.; Cronin, S. B. Nano Lett. 2012, 12, 4484-4489. PMID: 22889241.

(22) Mallorquí, A. D.; Alarcón-Lladó, E.; Mundet, I. C.; Kiani, A.; Demaurex, B.; de Wolf, S.; Menzel, A.; Zacharias, M.; Fontcuberta i Morral, A. Nano Res. 2015, 8, 673-681.

(23) Yuan, Y. R.; Pudensi, M. A. A.; Vawter, G. A.; Merz, J. L. J. Appl. Phys. 1985, 58, 397-403.

(24) Dhaka, V.; Oksanen, J.; Jiang, H.; Haggren, T.; Nykänen, A.; Sanatinia, R.; Kakko, J.-P.; Huhtio, T.; Mattila, M.; Ruokolainen, J.; Anand, S.; Kauppinen, E.; Lipsanen, H. Nano Lett. 2013, 13, 35813588 .

(25) Smith, L. M.; Jackson, H. E.; Yarrison-Rice, J. M.; Jagadish, C. Semicond. Sci. Technol. 2010, 25, 024010.

(26) Songmuang, R.; Giang, L. T. T.; Bleuse, J.; Hertog, M. D.; Niquet, Y. M.; Dang, L. S.; Mariette, H. Nano Lett. 2016, 16, 3426. PMID: 27081785.

(27) Hocevar, M.; Thanh Giang, L. T.; Songmuang, R.; den Hertog, M.; Besombes, L.; Bleuse, J.; Niquet, Y.-M.; Pelekanos, N. T. Appl. Phys. Lett. 2013, 102, 191103.

(28) Heiss, M.; et al. Nat. Mater. 2013, 12, 439.

(29) Mancini, L.; Fontana, Y.; Conesa-Boj, S.; Blum, I.; Vurpillot, F.; Francaviglia, L.; Russo-Averchi, E.; Heiss, M.; Arbiol, J.; Morral, A. F. i.; Rigutti, L. Appl. Phys. Lett. 2014, 105, 243106.

(30) Heiss, M.; Conesa-Boj, S.; Ren, J.; Tseng, H.-H.; Gali, A.; Rudolph, A.; Uccelli, E.; Peiró, F.; Morante, J. R.; Schuh, D.; Reiger, E.; Kaxiras, E.; Arbiol, J.; Fontcuberta i Morral, A. Phys. Rev. B: Condens. Matter Mater. Phys. 2011, 83, 045303.

(31) Plissard, S.; Dick, K. A.; Larrieu, G.; Godey, S.; Addad, A.; Wallart, X.; Caroff, P. Nanotechnology 2010, 21, 385602.

(32) Thelander, C.; Caroff, P.; Plissard, S.; Dey, A. W.; Dick, K. A. Nano Lett. 2011, 11, 2424-2429.

(33) de la Mata, M.; Magen, C.; Gazquez, J.; Utama, M. I. B.; Heiss, M.; Lopatin, S.; Furtmayr, F.; Fernández-Rojas, C. J.; Peng, B.; Morante, J. R.; Rurali, R.; Eickhoff, M.; Fontcuberta i Morral, A.; Xiong, Q.; Arbiol, J. Nano Lett. 2012, 12, 2579-2586. PMID: 22493937.

(34) Arbiol, J.; Estradé, S.; Prades, J. D.; Cirera, A.; Furtmayr, F.; Stark, C.; Laufer, A.; Stutzmann, M.; Eickhoff, M.; Gass, M. H.; Bleloch, A. L.; Peiró, F.; Morante, J. R. Nanotechnology 2009, 20, 145704.

(35) Shtrikman, H.; Popovitz-Biro, R.; Kretinin, A.; Houben, L.; Heiblum, M.; Bukala, M.; Galicka, M.; Buczko, R.; Kacman, P. Nano Lett. 2009, 9, 1506-1510. PMID: 19253998.

(36) Shtrikman, H.; Popovitz-Biro, R.; Kretinin, A.; Heiblum, M. Nano Lett. 2009, 9, 215-219.

(37) Plochocka, P.; Mitioglu, A. A.; Maude, D. K.; Rikken, G. L. J. A.; Granados del Aguila, A.; Christianen, P. C. M.; Kacman, P.; Shtrikman, H. Nano Lett. 2013, 13, 2442-2447.

(38) Yuan, X.; Caroff, P.; Wong-Leung, J.; Fu, L.; Tan, H. H.; Jagadish, C. Adv. Mater. 2015, 27, 6096-6103.

(39) Chi, C.-Y.; Chang, C.-C.; Hu, S.; Yeh, T.-W.; Cronin, S. B.; Dapkus, P. D. Nano Lett. 2013, 13, 2506-2515. PMID: 23634790.

(40) Arab, S.; Chi, C.-Y.; Shi, T.; Wang, Y.; Dapkus, D. P.; Jackson, H. E.; Smith, L. M.; Cronin, S. B. ACS Nano 2015, 9, 1336-1340. PMID: 25565000.

(41) Chang, C. C.; Chi, C. Y.; Chen, C. C.; Huang, N.; Arab, S.; Qiu, J.; Povinelli, M. L.; Dapkus, P. D.; Cronin, S. B. Nano Res. 2014, 7, 163-170.

(42) de la Mata, M.; Leturcq, R.; Plissard, S. R; Rolland, C.; Magén, C.; Arbiol, J.; Caroff, P. Nano Lett. 2016, 16, 825-833. PMID: 26733426.

(43) Tutuncuoglu, G.; de la Mata, M.; Deiana, D.; Potts, H.; Matteini, F.; Arbiol, J.; Fontcuberta i Morral, A. Nanoscale 2015, 7, 19453-19460.
(44) Heiß, M.; Riedlberger, E.; Spirkoska, D.; Bichler, M.; Abstreiter, G.; Fontcuberta i Morral, A. J. Cryst. Growth 2008, 310, 1049-1056.

(45) Woolf, D. A.; Sobiesierski, Z.; Westwood, D. I.; Williams, R. H. J. Appl. Phys. 1992, 71, 4908-4915.

(46) Heim, U.; Hiesinger, P. Phys. Status Solidi B 1974, 66, 461-470.

(47) Kunzel, H.; Ploog, K. Appl. Phys. Lett. 1980, 37, 416-418.

(48) Zhang, G.; Tateno, K.; Sanada, H.; Tawara, T.; Gotoh, H.; Nakano, H. Appl. Phys. Lett. 2009, 95, 123104.

(49) Ploog, K.; Döhler, G. H. Adv. Phys. 1983, 32, 285-359.

(50) van Weert, M. H. M.; Wunnicke, O.; Roest, A. L.; Eijkemans, T. J.; Yu Silov, A.; Haverkort, J. E. M.; 't Hooft, G. W.; Bakkers, E. P. A. M. Appl. Phys. Lett. 2006, 88, 043109.

(51) Signorello, G.; Karg, S.; Björk, M. T.; Gotsmann, B.; Riel, H. Nano Lett. 2013, 13, 917-924. PMID: 23237482.

(52) Oskooi, A. F.; Roundy, D.; Ibanescu, M.; Bermel, P.; Joannopoulos, J. D.; Johnson, S. G. Comput. Phys. Commun. 2010, $181,687-702$.

(53) Adachi, S. J. Appl. Phys. 1985, 58, R1-R29. 